\title{
Decomposição e liberação de nutrientes de coquetéis vegetais para utilização no Semiárido brasileiro ${ }^{1}$
}

\author{
The decomposition and release of nutrients by plant mixtures in the Brazilian semi- \\ arid regions
}

\author{
Vanderlise Giongo $^{2 *}$, Alessandra Monteiro Salviano Mendes ${ }^{3}$, Tony Jarbas Ferreira Cunha ${ }^{3}$ e Sandra Regina \\ Silva Galvão ${ }^{4}$
}

\begin{abstract}
Resumo - A decomposição e liberação de nutrientes de resíduos vegetais podem assumir importante papel no manejo da fertilidade dos solos da região semiárida. Portanto, o presente estudo teve por objetivo avaliar a taxa de decomposição da matéria seca (MS) e liberação de carbono (C) e nutrientes de coquetéis vegetais no Semiárido brasileiro. O experimento foi conduzido em casa de vegetação, no período de novembro de 2007 a junho de 2008, com coquetéis vegetais compostos por espécies de leguminosas (L) (calopogônio, Crotalaria juncea, Crotalaria spectabilis, feijão de porco, guandu, lab-lab) e não leguminosas (NL) (gergelim, girassol, mamona, milheto e sorgo), combinados nos seguintes tratamentos: T1 - 100\% NL; T2 - 100\% L; T3 - 75\% L e 25\% NL; T 4 - 50\% L e 50\% NL; T5 - 25\% L e 75\% NL. As taxas de decomposição da MS e liberação dos nutrientes foram monitoradas por meio de coleta dos resíduos, realizadas aos $8 ; 15 ; 30 ; 60 ; 90 ; 120 ; 150 ; 180 \mathrm{e}$ 210 dias após o início do experimento. Utilizou-se o delineamento experimental em blocos casualizados com três repetições. Os coquetéis apresentaram taxas de decomposição de MS e liberação de nutrientes semelhantes, exceto para $\mathrm{Ca}, \mathrm{Cu}$ e $\mathrm{Mn}$, em que os coquetéis compostos com predominância de espécies NL apresentaram liberação mais rápida. A partir da média dos valores de $k$ para todos os coquetéis estabeleceu-se a seguinte ordem de liberação de macronutrientes: $\mathrm{K}>\mathrm{N}>\mathrm{Ca}>\mathrm{Mg}>\mathrm{Pe}$ de micronutrientes: $\mathrm{Fe}>\mathrm{Mn}>\mathrm{Cu}>\mathrm{Zn}>\mathrm{B}$.
\end{abstract}

Palavras-chave - Adubação verde. Macronutrientes. Micronutrientes. Carbono orgânico. Taxa de mineralização.

\begin{abstract}
The decomposition and release of nutrients from plant residues can play an important role in the maintenance of soil fertility in semi-arid regions. Thus this study aimed to evaluate the rate of decomposition of dry matter (DM) and the release of carbon (C) and nutrients from plant mixtures used as green manure in the Brazilian Semi-arid regions. The experiment was carried out in a greenhouse, from November 2007 to July 2008, using plant mixtures made up of leguminous species (L) (calopo, Crotalaria juncea, Crotalaria spectabilis, jackbean, pigeon pea, lab-lab) and non-leguminous species (NL) (sesame, sunflower, castor, millet and sorghum) combined to give the following treatments: T1 - 100\% NL, T2 - 100\% L, T3 - 75\% $\mathrm{L}$ and $25 \% \mathrm{NL}, \mathrm{T} 4-50 \% \mathrm{~L}$ and $50 \% \mathrm{NL}, \mathrm{T} 5-25 \% \mathrm{~L}$ and $75 \% \mathrm{NL}$. The rates of decomposition and the release of nutrients were monitored through collection of the residue at $8 ; 15 ; 30 ; 60 ; 90 ; 120 ; 150 ; 180$ and 210 days after the beginning of the experiment. A randomized-block design was used, with three replications. The mixtures showed similar decomposition rates of DM and nutrient release, except for $\mathrm{Ca}, \mathrm{Cu}$ and $\mathrm{Mn}$, where the mixtures composed predominantly of NL species showed a higher release rate. From the average values of $k$ for all of the mixtures, it was possible to establish the following order of release of macronutrients: $\mathrm{K}>\mathrm{N}>\mathrm{Ca}>\mathrm{Mg}>\mathrm{P}$ and micronutrients: $\mathrm{Fe}>\mathrm{Mn}>\mathrm{Cu}>\mathrm{Zn}>\mathrm{B}$.
\end{abstract}

Key words - Green manure. Macronutrients. Micronutrients. Organic carbon. Mineralization rate.

\footnotetext{
*Autor para Correspondência

${ }^{1}$ Recebido para publicação em 21/01/2010; aprovado em 14/05/2011

Parte de pesquisa financiada pela EMBRAPA

${ }_{2}^{2}$ Pesquisadora da Embrapa Semiárido, Caixa Postal 23, 56.302-970, Petrolina-PE, vanderlise@cpatsa.embrapa.br, amendes@cpatsa.embrapa.br

3Pesquisador da Embrapa Semiárido, tony@cpatsa.embrapa.br

${ }^{4}$ Instituto Federal de Educação, Ciência e Tecnologia do Sertão Pernambucano/IFSertão-Campus Salgueiro, sandra.galvao@ifsertao-pe.edu.br
} 


\section{Introdução}

No Vale São Francisco, a produção de mangas é explorada por pequenos e grandes produtores com cultivo irrigado em diferentes tipos de solo (COSTA; TORNISIELO; REGITANO; 2008). No entanto, o cultivo intensivo do solo para produção de manga em associação às condições climáticas locais e o uso indiscriminado de insumos de fontes não renováveis favorece a degradação, principalmente pela intensificação dos processos de erosão e compactação, modificando os atributos físicos, químicos e biológicos do solo (AGUIAR; MONTEIRO, 2005; FERRACINI et al., 2001). Neste sentido, a adoção de novas tecnologias fundamentadas em bases conservacionistas, como o uso de cobertura vegetal, tem se tornado estratégia essencial para a recuperação e manutenção da qualidade dos solos (AZEVEDO et al., 2007; BAYER et al., 2001).

A utilização de coquetéis vegetais associados ao não revolvimento do solo pode se tornar estratégia de manejo viável para o cultivo de manga nos solos do Semiárido Tropical brasileiro. Nesses sistemas, espécies utilizadas como culturas de cobertura são semeadas em conjunto (misturadas) e quando atingem o estádio de pleno florescimento são cortadas e depositadas sobre o solo. Este manejo permite a movimentação dos nutrientes das camadas mais profundas do solo, extraídos por meio do sistema radicular, para a superfície, após o corte da fitomassa dos coquetéis e sua decomposição pela ação do ambiente. Além disso, serve como cobertura morta, reduzindo a perda de água do sistema e como fonte de material orgânico para o solo (BOER et al., 2007; DUDA et al., 2003). Porém a liberação de nutrientes desses coquetéis vegetais depende de diversos fatores: $(i)$ interação entre as espécies utilizadas; (ii) manejo da fitomassa; (iii) época de semeadura e corte; (iv) composição química do resíduo vegetal e relação $\mathrm{C} / \mathrm{N}$ ); e (v) condições edafoclimáticas (pluviosidade, aeração, temperatura, atividade macro e microbiológica do solo e do tipo de solo) (CRUSCIOL et al., 2008). Assim, no manejo de plantas de cobertura, a compreensão dos fatores que regulam a decomposição pode assumir importante papel no manejo das culturas, possibilitando a elaboração de técnicas de cultivo que melhorem a utilização de nutrientes contidos nos resíduos vegetais que formam a serapilheira (GAMARODRIGUES; GAMA-RODRIGUES; BRITO, 2007).

A utilização de culturas de cobertura objetiva gerar quantidades de matéria seca (MS) suficientes para manter o solo coberto durante todo o ano, aumentar o teor de matéria orgânica do solo, diminuir a evapotranspiração e consequentemente o processo de salinização é essencial para a sustentabilidade dos sistemas agrícolas em ambiente semiárido. Para isso, o conhecimento do tempo de permanência dos resíduos vegetais no ambiente e da dinâmica de liberação dos nutrientes presentes na sua fitomassa é de suma importância para o sucesso de um sistema sustentável de produção. O presente estudo teve como objetivo avaliar as taxas de decomposição e de liberação dos nutrientes de cinco coquetéis vegetais cultivados nas entrelinhas de mangueiras no Semiárido brasileiro.

\section{Material e métodos}

O trabalho foi realizado em casa de vegetação na EMBRAPA Semiárido, Petrolina-PE, no período de novembro de 2007 a junho de 2008. As unidades experimentais foram constituídas de vasos contendo $3,6 \mathrm{~kg}$ de solo. O solo utilizado foi o Argissolo Amarelo eutrófico (EMBRAPA, 2006) coletado em uma área sob caatinga preservada e apresenta os seguintes atributos na camada de 0-20 cm: 762,5 $\mathrm{g} \mathrm{kg}^{-1}$ de areia; $210,2 \mathrm{~g} \mathrm{~kg}^{-1}$ de silte; $27,3 \mathrm{~g} \mathrm{~kg}^{-1}$ de argila; $\mathrm{pH}\left(\mathrm{H}_{2} \mathrm{O}\right)=5,3$; matéria orgânica $(\mathrm{MO})=6,8 \mathrm{~g} \mathrm{~kg}^{-1} ; \mathrm{P}\left(\right.$ Mehlich 1) $=3,0 \mathrm{mg} \mathrm{dm}^{-3} ; \mathrm{H}+\mathrm{Al}=$ $1,81 \mathrm{cmol} \mathrm{dm}^{-3} ; \mathrm{K}^{+}=0,20 \mathrm{cmol} \mathrm{dm}_{\mathrm{c}}^{-3} ; \mathrm{Ca}^{2+}=1,0 \mathrm{cmol} \mathrm{dm}^{-3}$; $\mathrm{Mg}^{2+}=0,5 \mathrm{cmol}_{\mathrm{c}} \mathrm{dm}^{-3} ; \mathrm{Na}^{+}=0,02 \mathrm{cmol}_{\mathrm{c}} \mathrm{dm}^{-3}$; capacidade de troca catiônica $\left(\right.$ CTC) $=3,53 \mathrm{cmol}_{\mathrm{c}} \mathrm{dm}^{-3}$ e saturação por bases $(\mathrm{V})=49 \%$. As freqüências de irrigação adotadas para as unidades experimentais foram com intervalos de dois dias. Nas irrigações, a umidade do solo foi reajustada a $80 \%$ da capacidade de campo, com água destilada, sendo controlada por meio de pesagens diárias. Durante o período do experimento a temperatura e a umidade relativa do ar do interior da casa de vegetação foram monitoradas sendo suas médias de $32,8^{\circ} \mathrm{C}$ e $58,5 \%$, respectivamente.

Mediu-se a decomposição da fitomassa aérea de cinco coquetéis vegetais utilizados para adubação verde e cobertura do solo, plantadas em diferentes composições e proporções que constituíram os diferentes tratamentos (T): T1 - 100\% não leguminosas; T2 - 100\% leguminosas; T3 - 75\% leguminosas e $25 \%$ não leguminosas; T 4 - 50\% leguminosas e 50\% não leguminosas; T5 - 25\% leguminosas e $75 \%$ não leguminosas. Para composição dos coquetéis foram utilizadas onze espécies entre leguminosas e não-leguminosas (oleaginosas e gramíneas). As leguminosas utilizadas foram: calopogônio (Calopogonium mucunoide), Crotalaria juncea, Crotalaria spectabilis, feijão de porco (Canavalia ensiformes), guandu (Cajanus Cajan L.), lab-lab (DolichoslablabL.); easnão-leguminosas: gergelim(Sesamum indicum L.), girassol (Chrysantemum peruviamum), mamona (Ricinus communis L.), milheto (penissetum americanum L.) e sorgo (Sorghum vulgare Pers.).

A fitomassa aérea foi proveniente dos coquetéis vegetais que foram semeados no período chuvoso, janeiro a março de 2007, entre as fileiras da mangueira, a uma distância de 2,00 $\mathrm{m}$ do colo das plantas, em sulcos espaçados de $0,50 \times 0,50 \mathrm{~cm}$, em que cada parcela correspondia a uma área de $160 \mathrm{~m}^{2}(16 \times 10 \mathrm{~m})$. O plantio 
foi realizado semeando-se 16 linhas de coquetéis vegetais na entrelinha das mangueiras dentro de cada parcela. Para garantir a uniformidade de distribuição das sementes nas linhas, inicialmente foram distribuídas as sementes de menor tamanho, seguido pelas de tamanho intermediário e posteriormente as maiores, evitando-se assim o efeito de segregação. As quantidades de sementes utilizadas em cada tratamento são apresentadas na Tabela 1.

Para determinar a massa do tecido vegetal no início da decomposição (To), amostras da fitomassa foram secas em estufa a $65-75{ }^{\circ} \mathrm{C}$ até atingirem massa constante. Utilizaram-se, para cada tratamento, $25 \mathrm{~g}$ de fitomassa seca, seccionada em fragmentos de $5 \mathrm{~cm}$, sendo o material depositado diretamente sobre o solo dos vasos. A área de deposição dos vasos foi de $0,049 \mathrm{~m}^{2}$, portanto a quantidade aplicada de fitomassa vegetal sobre o vaso foi equivalente a $5.095 \mathrm{~kg} \mathrm{ha}^{-1}$.

O delineamento experimental foi em blocos completos casualizados, com cinco tratamentos e três repetições. Cada bloco foi constituído por 10 vasos (tempos de coleta) para cada tratamento, totalizando 50 vasos por bloco. Antes de iniciar o experimento foi realizada a análise química dos coquetéis vegetais (TAB. 2).

As coletas foram realizadas aos $8 ; 15 ; 30 ; 45 ; 60$; 90; 120; 150; 180 e 210 dias após o início do processo de decomposição, sendo coletado um vaso por tratamento, em cada bloco. O resíduo vegetal foi limpo manualmente, seco em estufa, à temperatura de $65^{\circ} \mathrm{C}$, até alcançar massa constante, moído e passado em peneira de $1 \mathrm{~mm}$ de malha.
Para as determinações de macro e micronutrientes, à exceção do $\mathrm{N}$ e do $\mathrm{B}$, o material vegetal foi submetido à digestão nítrico-perclórica conforme descrito em EMBRAPA (1999). Nesse extrato, a concentração de P foi determinada por espectrofotometria do metavanadato + molibdato, a de K por fotometria de emissão de chama, as de $\mathrm{Cu}, \mathrm{Zn}, \mathrm{Fe}, \mathrm{Mn}, \mathrm{Ca}$ e $\mathrm{Mg}$ por espectrofotometria de absorção atômica (EAA) com atomização em chama (ar-acetileno). Para a determinação de N, submeteu-se o material vegetal à digestão sulfúrica e posterior destilação do extrato pelo método micro-Kjeldahl. O extrato vegetal para determinação da concentração de $\mathrm{B}$ foi obtido pela dissolução das cinzas provenientes da incineração do material seco, com posterior determinação pelo método espectrofotométrico da azometina-H (EMBRAPA, 1999). O C foi determinado pelo método da perda de peso por ignição em mufla (KIHEL, 1985).

Os valores obtidos foram transformados em porcentagem relativa à massa e ao teor de nutrientes do início da decomposição (To). Com esses dados, foram determinadas as taxas de decomposição da biomassa e de liberação de nutrientes, para cada um dos tratamentos, utilizando-se o modelo matemático exponencial, $\mathrm{Mt}=\mathrm{Mi} \mathrm{e}^{-k \mathrm{~T}}$ (OLSON, 1963); sendo Mt os percentuais remanescentes de biomasa (MS), C e nutrientes após $T$ dias, e $\mathrm{Mi}=100 \%$, quando $T$ é igual a zero, ou seja no dia da deposição do material no solo. Obteve-se, assim, para cada tratamento, uma equação, sendo as mesmas ajustadas utilizando-se o programa SigmaPlot®10 (Systat Software Inc.). A partir do valor

Tabela 1 - Quantidade de sementes (g) das espécies plantadas em cada parcela, por tratamento

\begin{tabular}{|c|c|c|c|c|c|}
\hline Espécies & T1 & $\mathrm{T} 2$ & T3 & $\mathrm{T} 4$ & T5 \\
\hline \multicolumn{6}{|c|}{ Leguminosas } \\
\hline Calopogônio & 0,0 & 82,0 & 61,5 & 41,0 & 20,5 \\
\hline Crotalária juncea & 0,0 & 260,0 & 195,0 & 130,0 & 65,0 \\
\hline $\begin{array}{l}\text { Crotalária } \\
\text { spectabilis }\end{array}$ & 0,0 & 382,0 & 286,5 & 191,0 & 95,5 \\
\hline Feijão de porco & 0,0 & 4786,0 & 3589,5 & 2393,0 & 1196,5 \\
\hline Guandu & 0,0 & 272,0 & 204,0 & 136,0 & 68,0 \\
\hline Lab-lab & 0,0 & 1280,0 & 980,0 & 640,0 & 320,0 \\
\hline \multicolumn{6}{|c|}{ Não Leguminosas } \\
\hline Gergelim & 64,0 & 0,0 & 16,0 & 32,0 & 48,0 \\
\hline Girassol & 200,0 & 0,0 & 50,0 & 100,0 & 150,0 \\
\hline Mamona & 2210,0 & 0,0 & 552,5 & 1105,0 & 1657,5 \\
\hline Milheto & 64,0 & 0,0 & 16,0 & 32,0 & 48,0 \\
\hline Sorgo & 160,0 & 0,0 & 40,0 & 80,0 & 120,0 \\
\hline
\end{tabular}

\# T1 - 100\% não leguminosas; T2 - 100\% leguminosas; T3 - 75\% leguminosas e 25\% não leguminosas; T 4 - $50 \%$ leguminosas e $50 \%$ não leguminosas; T5 - 25\% leguminosas e 75\% não leguminosas 
Tabela 2 - Concentração de nutrientes (na massa seca) dos coquetéis vegetais utilizados no experimento

\begin{tabular}{|c|c|c|c|c|c|c|c|c|c|c|c|c|c|}
\hline \multirow{2}{*}{ Trat. } & $\mathrm{C}$ & $\mathrm{N}$ & $\mathrm{P}$ & $\mathrm{K}$ & $\mathrm{Ca}$ & $\mathrm{Mg}$ & $\mathrm{S}$ & B & $\mathrm{Cu}$ & $\mathrm{Fe}$ & $\mathrm{Mn}$ & $\mathrm{Zn}$ & $\mathrm{Na}$ \\
\hline & \multicolumn{7}{|c|}{ 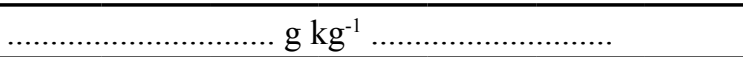 } & \multicolumn{6}{|c|}{ …............. $\mathrm{mg} \mathrm{kg}^{-1} \ldots \ldots \ldots \ldots \ldots \ldots \ldots$} \\
\hline $\mathrm{T} 1$ & 424 & 20,1 & 1,29 & 14,7 & 13,4 & 2,41 & 0,35 & 27 & 11,2 & 1227 & 115 & 29 & 169 \\
\hline $\mathrm{T} 2$ & 425 & 19,4 & 1,36 & 15,6 & 12,8 & 2,39 & 0,30 & 25 & 10,5 & 1210 & 123 & 38 & 122 \\
\hline $\mathrm{T} 3$ & 421 & 16,1 & 1,28 & 16,0 & 12,5 & 2,66 & 0,32 & 23 & 10,7 & 1180 & 129 & 34 & 115 \\
\hline $\mathrm{T} 4$ & 428 & 13,0 & 1,27 & 15,0 & 10,2 & 2,48 & 0,26 & 21 & 9,1 & 1173 & 107 & 37 & 125 \\
\hline T5 & 412 & 16,4 & 1,13 & 14,3 & 13,5 & 3,02 & 0,30 & 24 & 11,6 & 1187 & 145 & 34 & 102 \\
\hline
\end{tabular}

T1 - 100\% não leguminosas; T2 - 100\% leguminosas; T3 - 75\% leguminosas e 25\% não leguminosas; T 4 - $50 \%$ leguminosas e $50 \%$ não leguminosas; T5 - $25 \%$ leguminosas e $75 \%$ não leguminosas

de $k$ foi calculado o tempo necessário para a liberação de $50 \%$ (t50) e $95 \%$ ( $(95)$ dos nutrientes na MS dos coquetéis vegetais, sendo respectivamente: $\mathrm{t} 50=0,693 / k$ e $\mathrm{t} 95=3 / k$ (SHANKS; OLSON, 1961).

$\mathrm{Na}$ análise de variância das taxas de decomposição e liberação de nutrientes aplicou-se o teste $\mathrm{F}(\mathrm{P}<0,05)$. Quando F foi significativo, as médias foram comparadas pelo teste de Tukey $(\alpha=0,05)$. Essas análises estatísticas foram realizadas mediante uso do programa de computador SISVAR 4.6 (FERREIRA, 2003).

\section{Resultados e discussão}

Em todos os tratamentos, a cinética do processo de decomposição dos coquetéis vegetais apresentou um padrão semelhante, com uma fase inicial rápida seguida de outra mais lenta (TAB. 3). Entre 55 e 68 dias após o início do experimento, $50 \%$ da MS inicial dos coquetéis vegetais foi decomposta (TAB. 3). Após esse período, ocorreu uma fase de decomposição mais lenta, onde $95 \%$ da MS foi decomposta entre 240 a 294 dias após o início do experimento (TAB. 3).

A taxa de decomposição da MS e as constantes de liberação de C, N, P e K dos diferentes coquetéis vegetais não diferenciaram estatisticamente. Portanto, a taxa média de decomposição da MS foi de 0,0114 dia $^{-1}$ e as constantes de liberação de $\mathrm{C}$ e $\mathrm{N}$ foram de 0,0113 e 0,0104 dia $^{-1}$, respectivamente. Todavia, Dias et al. (2007) afirmam que muitos trabalhos têm sido realizados com a hipótese de que na mistura de gramíneas (C4) e leguminosas (C3), as plantas C4 são beneficiadas. Isso se deve, segundo os mesmos pesquisadores, à fixação do $\mathrm{N}_{2}$ atmosférico e excreção direta dos compostos nitrogenados liberados pelas raízes ou pela decomposição da liteira. Assim, parte do $\mathrm{N}$ necessário ao desenvolvimento e crescimento de uma gramínea forrageira pode ser adquirida com a introdução de leguminosa no sistema.

Em relação ao $\mathrm{C}$, considerando a produção de massa seca dos coquetéis vegetais, o tempo necessário para liberação de $50 \%$ foi de $56 ; 63 ; 67$; 64 e 58 dias, mineralizando 1.127 ; 794; 1.016; 935 e $875 \mathrm{~kg} \mathrm{ha}^{-1}$ de C para os tratamentos $\mathrm{T} 1$; T2; T3; T4 e T5, respectivamente (TAB. 4). Comportamento similar foi observado com nitrogênio, no qual os tratamentos T1; T2; T3; T4 e T5 necessitaram de 56; 63; 67; 64 e 58 dias, respectivamente, para liberarem, respectivamente, 57; 42; 37; 28 e $30 \mathrm{~kg} \mathrm{ha}^{-1}$ de N (TAB. 4). Segundo Gama-Rodrigues, Gama-Rodrigues e Brito (2007) isto ocorre porque o C se correlaciona positivamente com $\mathrm{N}$, indicando que a liberação desse nutriente acompanharia a perda de massa e, ou, a mineralização de C. O tratamento T1, apesar de

Tabela 3 - Produção e valores estimados da taxa de decomposição ( $k$ ) de massa seca (MS) dos coquetéis vegetais utilizados pelo modelo exponencial de primeira ordem e tempo necessário para decomposição de 50\% e 95\% do material depositado (dias)

\begin{tabular}{lccccc}
\hline \multicolumn{1}{c}{ Tratamento } & $\mathrm{Mg} \mathrm{ha}^{-1}$ & $\mathrm{k}\left(\mathrm{dia}^{-1}\right)$ & $\mathrm{t50}$ & $\mathrm{t95}$ & $\mathrm{R}^{2(1)}$ \\
\hline T1 - 100\% não leguminosas & 5,21 & $0,0125 \mathrm{a}$ & 55 & 240 & 0,92 \\
T2 - 100\% leguminosas & 3,78 & $0,0107 \mathrm{a}$ & 65 & 280 & 0,89 \\
T3 - 75\% leguminosas e 25\% não leguminosas & 4,73 & $0,0114 \mathrm{a}$ & 61 & 263 & 0,89 \\
T4 - 50\% leguminosas e 50\% não leguminosas & 4,29 & $0,0102 \mathrm{a}$ & 68 & 294 & 0,91 \\
T5 - 25\% leguminosas e 75\% não leguminosas & 4,17 & $0,0121 \mathrm{a}$ & 57 & 248 & 0,88 \\
\hline
\end{tabular}

Médias seguidas de letras iguais não diferem entre si pelo teste Tukey $(\alpha=0,05)$; ${ }^{(1)} \mathrm{P}<0,05$ 
Tabela 4 - Valores estimados da taxa de liberação $(k)$ de $\mathrm{C}$ e macronutrientes dos coquetéis vegetais utilizados pelo modelo exponencial de $1^{\text {a }}$ ordem e tempo necessário para liberação de $50 \%$ e $95 \%$ dos elementos presentes no material depositado (dias)

\begin{tabular}{|c|c|c|c|c|c|}
\hline Elemento & Tratamento & $\mathrm{k}\left(\mathrm{dia}^{-1}\right)$ & $\mathrm{t} 50$ & t95 & $\mathrm{R}^{2(1)}$ \\
\hline \multirow{5}{*}{$\mathrm{C}$} & T1 - 100\% não leguminosas & $0,0123 \mathrm{a}$ & 56 & 244 & 0,90 \\
\hline & $\mathrm{T} 2-100 \%$ leguminosas & $0,0110 \mathrm{a}$ & 63 & 273 & 0,83 \\
\hline & T3 - 75\% leguminosas e 25\% não leguminosas & $0,0103 \mathrm{a}$ & 67 & 291 & 0,84 \\
\hline & T4 - 50\% leguminosas e 50\% não leguminosas & $0,0109 \mathrm{a}$ & 64 & 275 & 0,84 \\
\hline & T5 - 25\% leguminosas e $75 \%$ não leguminosas & $0,0120 \mathrm{a}$ & 58 & 250 & 0,85 \\
\hline \multirow{5}{*}{$\mathrm{N}$} & T1 - 100\% não leguminosas & $0,0120 \mathrm{a}$ & 58 & 250 & 0,92 \\
\hline & $\mathrm{T} 2-100 \%$ leguminosas & $0,0105 \mathrm{a}$ & 66 & 286 & 0,91 \\
\hline & T3 - 75\% leguminosas e $25 \%$ não leguminosas & $0,0097 \mathrm{a}$ & 71 & 309 & 0,83 \\
\hline & T4 - 50\% leguminosas e 50\% não leguminosas & $0,0088 \mathrm{a}$ & 79 & 341 & 0,83 \\
\hline & T5 - 25\% leguminosas e $75 \%$ não leguminosas & $0,0109 \mathrm{a}$ & 64 & 275 & 0,85 \\
\hline \multirow{5}{*}{$\mathrm{P}$} & T1 - 100\% não leguminosas & $0,0088 \mathrm{a}$ & 79 & 341 & 0,73 \\
\hline & $\mathrm{T} 2-100 \%$ leguminosas & $0,0072 \mathrm{a}$ & 96 & 417 & 0,70 \\
\hline & T3 - 75\% leguminosas e $25 \%$ não leguminosas & $0,0074 \mathrm{a}$ & 94 & 405 & 0,59 \\
\hline & T4 - 50\% leguminosas e $50 \%$ não leguminosas & 0,0089 a & 78 & 337 & 0,61 \\
\hline & T5 - 25\% leguminosas e $75 \%$ não leguminosas & $0,0075 \mathrm{a}$ & 92 & 400 & 0,61 \\
\hline \multirow{5}{*}{$\mathrm{K}$} & $\mathrm{T} 1-100 \%$ não leguminosas & $0,0147 \mathrm{a}$ & 47 & 204 & 0,83 \\
\hline & $\mathrm{T} 2-100 \%$ leguminosas & $0,0137 \mathrm{a}$ & 51 & 219 & 0,93 \\
\hline & T3 - 75\% leguminosas e $25 \%$ não leguminosas & $0,0146 \mathrm{a}$ & 47 & 205 & 0,81 \\
\hline & T4 - 50\% leguminosas e 50\% não leguminosas & $0,0131 \mathrm{a}$ & 53 & 229 & 0,79 \\
\hline & T5 - 25\% leguminosas e $75 \%$ não leguminosas & $0,0141 \mathrm{a}$ & 49 & 213 & 0,80 \\
\hline \multirow{5}{*}{$\mathrm{Ca}$} & T1 - 100\% não leguminosas & $0,0104 \mathrm{a}$ & 67 & 288 & 0,84 \\
\hline & $\mathrm{T} 2-100 \%$ leguminosas & $0,0080 \mathrm{~b}$ & 87 & 375 & 0,74 \\
\hline & T3 - 75\% leguminosas e $25 \%$ não leguminosas & $0,0086 \mathrm{~b}$ & 81 & 349 & 0,78 \\
\hline & T4 - 50\% leguminosas e 50\% não leguminosas & $0,0084 \mathrm{~b}$ & 83 & 357 & 0,77 \\
\hline & T5 - 25\% leguminosas e $75 \%$ não leguminosas & $0,0094 \mathrm{ab}$ & 74 & 319 & 0,75 \\
\hline \multirow{5}{*}{$\mathrm{Mg}$} & T1 - 100\% não leguminosas & $0,0096 \mathrm{a}$ & 72 & 313 & 0,84 \\
\hline & $\mathrm{T} 2-100 \%$ leguminosas & $0,0080 \mathrm{a}$ & 87 & 375 & 0,82 \\
\hline & T3 - 75\% leguminosas e $25 \%$ não leguminosas & $0,0092 \mathrm{a}$ & 75 & 326 & 0,80 \\
\hline & T4 - 50\% leguminosas e 50\% não leguminosas & $0,0072 \mathrm{a}$ & 96 & 417 & 0,74 \\
\hline & T5 - 25\% leguminosas e $75 \%$ não leguminosas & $0,0103 \mathrm{a}$ & 67 & 291 & 0,83 \\
\hline
\end{tabular}

Médias seguidas de letras iguais, na mesma coluna, não diferem entre si pelo teste Tukey $(\alpha=0,05) ;{ }^{(1)} \mathrm{P}<0,05$

possuir uma mineralização mais rápida, liberou para o $30 \mathrm{~s}$ coquetéis vegetais com não leguminosas tem somente a capacidade recicladora deste elemento.

Os coquetéis vegetais não diferiram entre si quanto às constantes de liberação para a maioria dos macronutrientes $(\mathrm{N}, \mathrm{K}, \mathrm{P}$ e $\mathrm{Mg}$ ), porém os dados não comparam quantidades adicionadas dos elementos, apenas sua dinâmica da liberação. Resultado diferente foi observado para o $\mathrm{Ca}$ (TAB. 4). Os coquetéis vegetais compostos com uma maior proporção de espécies 
Tabela 5 - Valores estimados da taxa de liberação $(k)$ de nutrientes dos coquetéis vegetais utilizados pelo modelo exponencial de $1^{\text {a }}$ ordem e tempo necessário para liberação de $50 \%$ e $95 \%$ dos micronutrientes presentes no material depositado (dias)

\begin{tabular}{|c|c|c|c|c|c|}
\hline Nutriente & Tratamento & $\mathrm{k}\left(\mathrm{dia}^{-1}\right)$ & $\mathrm{t} 50$ & T95 & $\mathrm{R}^{2(1)}$ \\
\hline \multirow{5}{*}{ B } & T1 - $100 \%$ não leguminosas & $0,0113 \mathrm{a}$ & 61 & 265 & 0,92 \\
\hline & $\mathrm{T} 2-100 \%$ leguminosas & $0,0095 \mathrm{a}$ & 73 & 316 & 0,89 \\
\hline & T3 - 75\% leguminosas e $25 \%$ não leguminosas & $0,0097 \mathrm{a}$ & 71 & 309 & 0,90 \\
\hline & $\mathrm{T} 4$ - $50 \%$ leguminosas e $50 \%$ não leguminosas & $0,0081 \mathrm{a}$ & 86 & 370 & 0,87 \\
\hline & T5 - $25 \%$ leguminosas e $75 \%$ não leguminosas & $0,0111 \mathrm{a}$ & 62 & 270 & 0,88 \\
\hline \multirow{5}{*}{$\mathrm{Cu}$} & T1 - $100 \%$ não leguminosas & $0,0120 \mathrm{ab}$ & 58 & 250 & 0,92 \\
\hline & $\mathrm{T} 2$ - $100 \%$ leguminosas & $0,0090 \mathrm{~b}$ & 77 & 333 & 0,76 \\
\hline & $\mathrm{T} 3$ - $75 \%$ leguminosas e $25 \%$ não leguminosas & $0,0138 \mathrm{a}$ & 50 & 217 & 0,78 \\
\hline & $\mathrm{T} 4$ - $50 \%$ leguminosas e $50 \%$ não leguminosas & $0,0096 \mathrm{ab}$ & 75 & 326 & 0,91 \\
\hline & T5 - $25 \%$ leguminosas e $75 \%$ não leguminosas & $0,0132 \mathrm{a}$ & 53 & 227 & 0,88 \\
\hline \multirow{5}{*}{$\mathrm{Zn}$} & T1 - $100 \%$ não leguminosas & $0,0107 \mathrm{a}$ & 65 & 280 & 0,87 \\
\hline & $\mathrm{T} 2-100 \%$ leguminosas & $0,0102 \mathrm{a}$ & 68 & 294 & 0,87 \\
\hline & T3 - $75 \%$ leguminosas e $25 \%$ não leguminosas & $0,0104 \mathrm{a}$ & 67 & 288 & 0,87 \\
\hline & $\mathrm{T} 4$ - $50 \%$ leguminosas e $50 \%$ não leguminosas & $0,0095 \mathrm{a}$ & 73 & 316 & 0,92 \\
\hline & T5 - $25 \%$ leguminosas e $75 \%$ não leguminosas & $0,0111 \mathrm{a}$ & 62 & 270 & 0,85 \\
\hline \multirow{5}{*}{$\mathrm{Fe}$} & T1 - $100 \%$ não leguminosas & $0,0149 \mathrm{a}$ & 47 & 201 & 0,87 \\
\hline & $\mathrm{T} 2-100 \%$ leguminosas & $0,0132 \mathrm{a}$ & 53 & 227 & 0,83 \\
\hline & $\mathrm{T} 3$ - $75 \%$ leguminosas e $25 \%$ não leguminosas & $0,0145 \mathrm{a}$ & 48 & 207 & 0,81 \\
\hline & $\mathrm{T} 4$ - $50 \%$ leguminosas e $50 \%$ não leguminosas & $0,0124 \mathrm{a}$ & 56 & 242 & 0,84 \\
\hline & T5 - $25 \%$ leguminosas e $75 \%$ não leguminosas & $0,0142 \mathrm{a}$ & 49 & 211 & 0,83 \\
\hline \multirow{5}{*}{$\mathrm{Mn}$} & T1 - $100 \%$ não leguminosas & $0,0133 \mathrm{ab}$ & 52 & 226 & 0,88 \\
\hline & $\mathrm{T} 2-100 \%$ leguminosas & $0,0125 \mathrm{ab}$ & 55 & 240 & 0,87 \\
\hline & $\mathrm{T} 3$ - $75 \%$ leguminosas e $25 \%$ não leguminosas & $0,0122 \mathrm{ab}$ & 57 & 246 & 0,85 \\
\hline & $\mathrm{T} 4$ - $50 \%$ leguminosas e $50 \%$ não leguminosas & $0,0098 \mathrm{~b}$ & 71 & 306 & 0,83 \\
\hline & T5 - $25 \%$ leguminosas e $75 \%$ não leguminosas & $0,0150 \mathrm{a}$ & 46 & 200 & 0,86 \\
\hline
\end{tabular}

Médias seguidas de letras iguais, na mesma coluna, não diferem entre si pelo teste Tukey $(\alpha=0,05)$; ${ }^{(1)} \mathrm{P}<0,05$

não leguminosas (100 e 75\%) apresentaram as maiores constantes de liberação para esse nutriente; consequentemente, mineralização mais rápida, conforme pode ser observado no t50 e 195 para esses tratamentos (TAB. 4).

Dentre os macronutrientes, o $\mathrm{K}$ foi o nutriente liberado mais rapidamente, com tempo de meia-vida de 49 dias, em média, e o $\mathrm{P}$ o que necessitou de mais tempo para a liberação de $50 \%$, com média de 88 dias. Do mesmo modo, para que aproximadamente 95\% do $\mathrm{K}$ e $\mathrm{P}$ contidos nos coquetéis fossem liberados, o tempo máximo obtido foi de 214 e 380 dias, em média, respectivamente. A maior velocidade de liberação do $\mathrm{K}$, em relação aos demais em estudo, pode ser atribuída ao fato de o $\mathrm{K}$ ser um elemento que não está associado a nenhum componente estrutural do tecido vegetal, e encontrar-se na forma iônica (MEURER, 2006; ROSSI et al., 2008; TORRES; PEREIRA, 2008). Por essa razão, ele pode ser facilmente extraído dos tecidos das plantas, tanto pela água da chuva quanto pela própria umidade do solo (CALONEGO; FOLONI; ROSOLEM, 2005). Por 
outro lado todos esses índices demonstram que o P é o elemento mais lento para ser liberado.

A partir da média dos valores de $k$ para todos os coquetéis vegetais, tornou-se possível estabelecer a seguinte ordem de liberação para os macronutrientes: $\mathrm{K}>\mathrm{N}>\mathrm{Ca}>\mathrm{Mg}>\mathrm{P}$, cujo os tempos médios de meia vida foram, respectivamente, $49 ; 68 ; 78 ; 79$ e 88 e os tempos, para que cerca de $95 \%$ dos nutrientes contidos nos coquetéis fossem liberados foram de $214 ; 292 ; 338$; 344 e 380 dias. Assim, as distintas taxas de liberação de nutrientes dos coquetéis vegetais avaliados no presente estudo mostraram a necessidade de estudos futuros do manejo de resíduos vegetais como fonte de nutrientes para a cultura principal.

Para os micronutrientes, os coquetéis vegetais também apresentaram constantes de liberação de nutrientes semelhantes, exceto para o $\mathrm{Cu}$ e o $\mathrm{Mn}$. Os coquetéis vegetais compostos com maior proporção de espécies não leguminosas (100 e 75\%) apresentaram as maiores constantes de liberação para os micronutrientes $\mathrm{Cu}$ e Mn, e, consequentemente, mineralização mais rápida conforme pode ser observado no tempo de meia-vida dos nutrientes para esse tratamento (TAB. 5). Nota-se que o resíduo do $\mathrm{T} 3$ apresentou comportamento intermediário em relação às outras coberturas vegetais (TAB. 5).

Levando-se em consideração os tempos de meia-vida observados no presente estudo, o Fe foi o nutriente liberado mais rapidamente, com 50 dias, em média, e o $\mathrm{B}$ o que necessitou de mais tempo para a liberação, com média de 71 dias.

Em todos os tratamentos, o B foi o micronutriente liberado mais lentamente, principalmente no coquetel composto por $50 \%$ de espécies leguminosas e $50 \%$ de não leguminosas, onde foram estimados os tempos necessários para liberação, em 86 e 370 dias, de 50 e 95\% da sua quantidade total. Isso se deve, provavelmente, ao papel estrutural, semelhante ao cálcio, que este elemento exerce na planta, fazendo ligações pécticas na parede celular. O boro, especificamente, contribui na estabilidade da parede e da membrana celular, ligando dois polissacarídeos pectínicos chamados de Rhamnogalacturona II (RGII), sendo este complexo encontrado em 22 espécies de plantas (MATOH; KAWAGUCHI; KOBAYASHI, 1996). Assim, sua liberação está mais fortemente relacionada com o processo de mineralização, tornando-a mais lenta, quando comparada a elementos não-estruturais, como o $\mathrm{K}$ que pode ser transferido para o solo por lixiviação.

\section{Conclusões}

1.Os coquetéis vegetais estudados apresentaram taxas de decomposição e de liberação de nutrientes similares para C, N, P, K, Mg, Zn, Fe e B, mas não para $\mathrm{Ca}, \mathrm{Cu}$ e Mn;
2.A partir da média dos valores de $k$ para todos os coquetéis vegetais, foi estabelecida a seguinte ordem de liberação respectivamente de macro e micronutrientes: $\mathrm{K}>\mathrm{N}>\mathrm{Ca}>\mathrm{Mg}>\mathrm{P}$ e $\mathrm{Fe}>\mathrm{Mn}>\mathrm{Cu}>\mathrm{Zn}>\mathrm{B}$.

\section{Agradecimento}

Ao Conselho Nacional de Desenvolvimento Científico e Tecnológico (CNPq), pela concessão de bolsa de iniciação científica.

\section{Referências}

AGUIAR, T. J. A.; MONTEIRO, M. S. L. Modelo agrícola e desenvolvimento sustentável: a ocupação do Cerrado piauiense. Ambiente e Sociedade, v. 08, n. 02, p. 1-18, 2005.

AZEVEDO, D. M. P. et al. Atributos físicos e químicos de um Latossolo Amarelo e distribuição do sistema radicular da soja sob diferentes sistemas de preparo no cerrado maranhense. Revista Ciência Agronômica, v. 38, n. 01, p. 32-40, 2007.

BAYER, C. et al. Changes in soil organic matter fractions under subtropical no-till cropping systems. Soil Science Society of America Journal, v. 65, n. 05, p. 1473-1478, 2001.

BOER, C. A. et al. Ciclagem de nutrientes por plantas de cobertura na entressafra em um solo de cerrado. Pesquisa Agropecuária Brasileira, v. 42, n. 09, p. 1269-1276, 2007.

CALONEGO, J. C.; FOLONI, J. S. S.; ROSOLEM, C. A. Lixiviação de potássio da palha de plantas de cobertura em diferentes estádios de senescência após dessecação química. Revista Brasileira de Ciência do Solo, v. 29, n. 01, p. 99-108, 2005.

COSTA, M. A.; TORNISIELO, V. L.; REGITANO, J. B. Mobilidade do paclobutrazol em um solo franco-arenoso cultivado com manga no nordeste brasileiro. Revista Brasileira de Ciência do Solo, Viçosa , v. 32, n. 5, p. 2177-2182 2008.

CRUSCIOL, C. A. C. et al. Taxas de decomposição e de liberação de macronutrientes da palhada de aveia preta em plantio direto. Bragantia, v. 67, n. 02, p. 261-266, 2008.

DIAS, P. F. et al. Transferência do $\mathrm{N}$ fixado por leguminosas arbóreas para o capim Survenola crescido em consórcio. Ciência Rural, v. 37, n. 02, p. 352-356, 2007.

DUDA, G. P. et al. Perennial herbaceous legumes as live soil mulches and their effects on $\mathrm{C}, \mathrm{N}$ and $\mathrm{P}$ of the microbiol biomass. Scientia Agricola, v. 60, n. 01, p. 139-147, 2003.

EMPRESA BRASILEIRA DE PESQUISA AGROPECUÁRIA (EMBRAPA). Centro Nacional de Pesquisa Agropecuária de Solos. Sistema Brasileiro de Classificação de Solos. 2. ed. Brasília: EMBRAPA/CNPTIA, 2006. 412 p.

EMPRESA BRASILEIRA DE PESQUISA AGROPECUÁRIA (EMBRAPA). Centro Nacional de Pesquisa Informática Agropecuária. Manual de análises químicas de solos, plantas e fertilizantes. Brasília: Embrapa/CNPTIA, 1999. 370 p. 
FERRACINI, V. L. et al. Análise de risco de contaminação das águas subterrâneas e superficiais da região de Petrolina (PE) e Juazeiro (BA). Pesticidas: Revista de Ecotoxicologia e Meio Ambiente, v. 11, n. 01, p. 1-16, 2001.

FERREIRA, D. F. Programa SISVAR (Sistema de Análise de Variância): versão 4,6 (Build 6,0). Lavras: UFLA/DEX, 2003.

GAMA-RODRIGUES, A. C.; GAMA-RODRIGUES, E. F.; BRITO, E. C. Decomposição e liberação de nutrientes de resíduos culturais de plantas de cobertura em argissolo vermelhoamarelo na região noroeste Fluminense (RJ). Revista Brasileira de Ciência do Solo, v. 31, n. 06, p. 1421-1428, 2007.

KIEHL, J. E. Fertilizantes orgânicos: análise de fertilizantes orgânicos. Campinas: Agronômica Ceres, 1985. 492 p.

MATOH, T.; KAWAGUCHI, S.; KOBAYASHI, M. Ubiquity of a borate-rhamnogalacturonan II complex in the cell walls of higher plants. Plant Cell Physiology, v. 37, n. 04, p. 636640, 1996.
MEURER, E. J. Potássio. In: FERNANDES, M. S. Nutrição mineral de plantas. Viçosa: UFV, 2006. Cap. 11, p. 281-298.

OLSON, J. S. Energy storage and the balance of producers and decomposers in ecological systems. Ecology, v. 44, n. 02, p. 322-331, 1963.

ROSSI, C. Q. et al. Liberação de macronutrientes de resíduos do consórcio entre mucuna preta e milho sob sistema orgânico de produção. Revista Universidade Rural, v. 28, n. 02, p. 01-10, 2008.

SHANKS, R.; OLSON, J. S. First year breakdown of leaf litter in Southern Appalachia Forest. Science, v. 134, n. 3473, p. 194-195, 1961.

TORRES, J. L. R.; PEREIRA, M. G. Dinâmica do potássio nos resíduos vegetais de plantas de cobertura no Cerrado. Revista Brasileira de Ciência do Solo, v. 32, n. 04, p. 1609-1618, 2008. 